\title{
Design and Analysis of a Novel Coupled Mechanism Based on a Spliced Polyhedron with Two Regular Tetrahedrons
}

\author{
Huifang $\mathrm{Gao}^{1}$ and Jingfang $\mathrm{Liu}^{2, *}$ \\ College of Mechanical Engineering and Applied Electronics Technology, Beijing \\ University of Technology, Beijing 100124, China \\ 'gaohuifang369@126.com, ${ }^{2} j f l i u @ b j u t . e d u . c n$ \\ * Corresponding Author
}

\begin{abstract}
Traditional methods and theories on designing parallel mechanisms are not suitable for related researches on hybrid mechanisms due to complicated connecting chains. The situation hampers the development of coupling mechanisms. Polyhedrons with fascinating appearance and geometry provide many choices for coupled inventions. Based on the compounds of two regular tetrahedrons, an equivalent basic geometrical model is determined by designing three substitutive parts first. These substitutions are interconnected with revolution joints (R-pairs) only. Then a loop linkage corresponding to a face of the spliced polyhedron is extracted and modified to be a movable elementary unit. Finally, the coupled model with one degree of freedom (DOF) can be synthesized symmetrically with six movable elementary loops. To verify the rationality and motion properties of the coupled structure, its mobility is analyzed with the independent motion shunting measurement and motion simulations are implemented. The results demonstrate the potential application of the novel system as an executing manipulator for mobile robots.
\end{abstract}

Keywords: spliced polyhedron, substitutive parts, reciprocal motion, adding chains

\section{Introduction}

Mechanical innovation has always been a hot topic of mechanism design [1]. Many practical hybrid manipulators [2-5] have been applied in some fields. Coupling mechanism is a type of more complicated hybrid structure due to the dependent motion chains between the base and the end-effector. Many coupled structures, such as the retractable canvas, network antenna for Space Station and magical Hoberman SwitchPitch Ball, have been used to in certain workplace to meet higher requirements. However, the traditional methods and theories for synthesizing parallel mechanisms [6-10] are not appropriate for the coupled structures.

Polyhedral structures are characterized with attractive appearance and geometry and occurring naturally. Polyhedron has been used in several areas of mathematics, chemistry, architecture, mechanism, and other scientific fields. To investigate more innovative mechanisms, many researchers have been drawn to the researches on designing coupled mechanism based on various polyhedra. This can be traced back to an octahedral structure invented by Fuller in 1948. Then you and Pellegrino [10] proposed a two-dimensional foldable linkage and indicated the adopted approach could be extendable to threedimensional dome constructions. Laliberté and Gosselin [11] proposed a mechanical arrangement based on a single type of component for different polyhedral constructions. Ref [12] presented a polyhedral rolling machine by means of combinations of a set of prismatic pairs, parallelogram scaling units and revolution pairs. For extensive range and superior performance of polyhedral coupled mechanisms, many researchers also had conducted in-depth theoretical studies on synthesis approaches and mobility analysis of 
coupled mechanisms [13-15]. Dai and Jones designed new developable mechanisms according to the origami and handicraft in 1999 [16]. Then Dai et al. investigated a scalable mechanism and analyzed its mobility by decomposing the mechanism into some elementary parallel mechanisms with single DOF based on the screw theory [17]. You [18] investigated the motion characteristics of the Hoberman sphere and pointed out its potential application in 2007. Liu et al. [19-20] proposed a new mobility method for coupled mechanisms by converting a complicated mechanism into a simple parallel structure with several equivalent generalized chains based on the screw theory. The method is practical for complex coupled mechanisms, especially symmetrical systems with multi-loop limbs.

In this paper a novel symmetrical coupled mechanism based on a spliced polyhedral compound of two regular tetrahedrons is proposed. By designing three types of substitutive components, the equivalent basic model is connected first with R-pairs only. Based on the screw theory the polyhedral coupled model is established with movable elementary loops by adding links in Section 2. Then the mobility of the novel mechanism is analyzed to identify the motion and symmetry properties in Section 3. The corresponding simulations are implemented and demonstrate the validity of the mechanism in Section 4. Some results and discussion are conducted in Section 5 and related conclusions are drawn in the last section.

\section{Design of the Novel Coupled Mechanism}

Regular tetrahedron is the simplest one of all five Platonic polyhedrons. It is particular that the distance between any two vertexes of a regular tetrahedron is equal and the number of faces meeting at every vertex is same. The compound of two regular tetrahedrons is selected as the fundamental structure here. First three types of substitutive components for vertexes and edges are designed according to the merged condition at each vertex. The equivalent basic construction can be modeled by connecting corresponding substitutions with R-pairs only. To obtain the expected motion, the mobility of one loop linkage corresponding to a face of the compound is analyzed and constraint sets are modified by adding some chains symmetrically based on the screw theory. At last each movable elementary unit is arranged into corresponding face location for the novel coupled model.

\subsection{Establishment of the Equivalent Basic Model}

As shown in Figure 1, the sketch of the polyhedron is a spatial solid bounded by six identical triangles containing five vertexes and nine edges. There are four triangles meeting at vertexes $B\left(B^{\prime}\right), C\left(C^{\prime}\right)$ and $D\left(D^{\prime}\right)$ and three triangles merging at $A$ and $A^{\prime}$. Based on the geometrical connections, three kinds of substitutive parts are designed and shown in Figure 2 respectively.

Vertexes $B\left(B^{\prime}\right), C\left(C^{\prime}\right)$ and $D\left(D^{\prime}\right)$ are substituted by square nodes with four R-pairs in Figure 2a. Vertexes $A$ and $A^{\prime}$ are replaced by regular triangles with three R-pairs in Figure $2 \mathrm{~b}$. Each edge in Figure 1 is represented by a binary link with two parallel R-pairs at both ends in Figure 2c. The three types of substitutions are connected to form the equivalent basic model shown in Figure 3. 


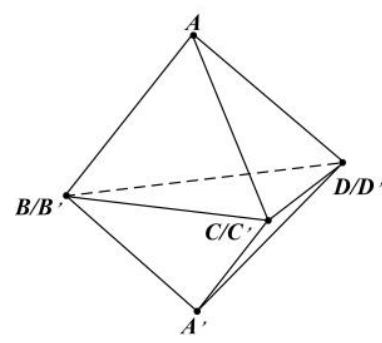

Figure 1. Sketch of the Compound of Two Regular Tetrahedrons

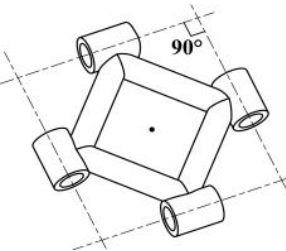

(a) Square Node Part

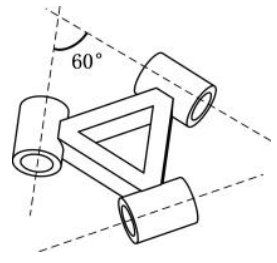

(b) Regular Triangular Part

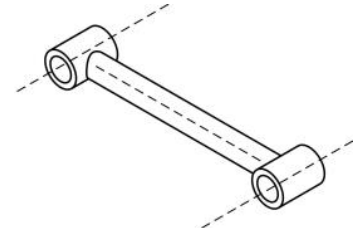

(c) Binary Link

Figure 2. Substitutive Components of Vertexes and Edges

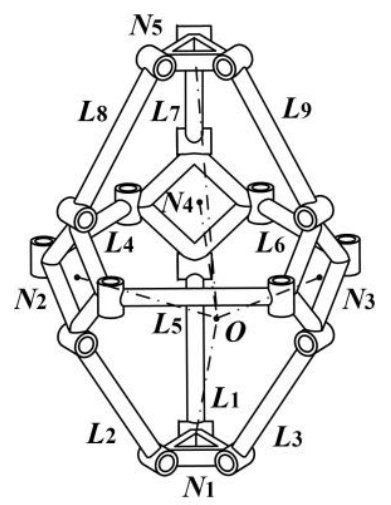

Figure 3. Equivalent Basic Model

\subsection{Construction of the Movable Elementary Unit}

As illustrated in Figure 3, the equivalent basic model contains six identical 6-bar linkages located in the face location of polyhedral compound. Due to the triangular faces, the compound framework is usually constrained. Since each loop is restricted with same screw constraints, it is feasible to simplify the related analysis of the entire complicated mechanism to be that of the 6-bar linkage. Hence, the primary issue is to determine the movability of a 6-bar loop. Taking one loop $N_{1}-L_{2}-N_{2}-L_{5}-N_{3}-L_{3}-N_{1}$ in Figure 4 as an example, the linkage matches up with the triangular face $A^{\prime} B^{\prime} C^{\prime}$ in Figure 1 . The constraint screw system of the 6-bar loop is analyzed first. 


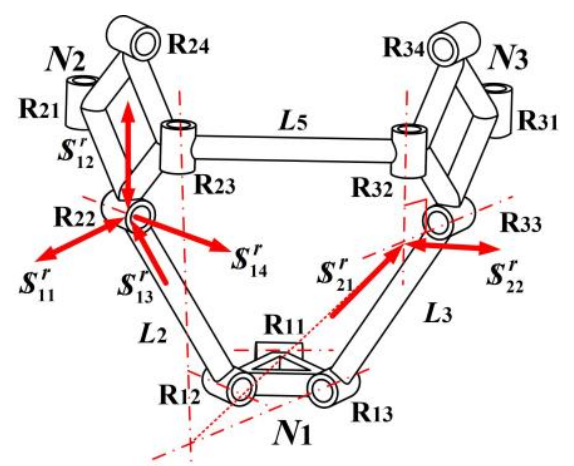

Figure 4. Construction and Constraints of a 6-Bar Loop

The constraint screw system of the original loop in Figure 4 is analyzed first. Taking $N_{1}$ as the base and $N_{2}$ as the end, the loop can be decomposed to two parallel branches, i.e. branch 1 labeled as $N_{1}-\mathrm{R}_{12}-\mathrm{R}_{22}-N_{2^{-}}$and branch 2 labeled as $N_{1}-\mathrm{R}_{13}-\mathrm{R}_{33}-N_{3}-\mathrm{R}_{32}-\mathrm{R}_{23^{-}} N_{2^{-}}$, respectively. The constraint sets for $N_{2}$ from the above two parallel branches are marked by red arrows. Constraints of branch 1 include two constraint couples and two constraint forces. The direction of constraint couples $\boldsymbol{S}_{11}^{r}$ and $\boldsymbol{S}_{12}^{r}$ is perpendicular to $\mathrm{R}_{22}$-axis. One constraint force $\boldsymbol{S}_{13}^{r}$ is along the direction of the link $L_{2}$ and the other one $\boldsymbol{S}_{14}^{r}$ is parallel to $\mathrm{R}_{22}$-axis. Constraints of branch 2 contain a constraint force and a constraint couple. The direction of the constraint force $\boldsymbol{S}_{21}^{r}$ is along the line defined by two intersection points, one determined by the $\mathrm{R}_{32^{-}}$and $\mathrm{R}_{33^{-}}$-axes, and the other by $\mathrm{R}_{23^{-}}$and $\mathrm{R}_{13}$-axes. The direction of the couple $\boldsymbol{S}_{22}^{r}$ is normal to the plane defined by $\mathrm{R}_{32}$-and $\mathrm{R}_{33}$-axes. Therefore, the 6-bar loop in Figure 4 is constrained.

To obtain an movable elementary unit, the approach of adding chains are adopt to modify the constraint sets of the two branches based on the reciprocity and relativity of screws [21]. The requirements of reciprocal condition for a given screw system is summarized in Table 1 . The sign $S_{i}(i=1,2)$ denotes the motion screw in a known screw system $\boldsymbol{S}$ and $\boldsymbol{S}_{i}^{r}(i=1,2)$ represents the reciprocal constraint screw in $\boldsymbol{S}$. The condition $i=1$ is the line vector and $i=2$ is the couple.

Tabel 1. Reciprocal Condition for Constraint Screws of a Known Screw System $S$

\begin{tabular}{|c|c|c|}
\hline Numble & $\begin{array}{c}\text { Demanded constraint screws } \\
\text { for } \boldsymbol{S}\end{array}$ & Reciprocal condition \\
\hline 1 & line vector $\boldsymbol{S}_{1}^{r}$ & $\boldsymbol{S}_{1}^{r}$ must be perpendicular to all \\
$\boldsymbol{S}_{2}$ and intersect with all $\boldsymbol{S}_{1}$ \\
\hline 2 & couple $\boldsymbol{S}_{2}^{r}$ & $\boldsymbol{S}_{2}^{r}$ must be perpendicular to all $\boldsymbol{S}_{1}$ \\
\hline
\end{tabular}

One simple way of adding a binary link is used to eliminate a constrain force. The part shown in Figure 2c is connected to $L_{2}$ in branch 1 for a new linkage group shown in Figure 5. This can eliminate the constraint force $\boldsymbol{S}_{13}^{r}$ and obtain the translational motion perpendicular to $R_{12^{-}}, R_{2^{-}}$and $R_{22^{-}}$axes simultaneously. By adding links symmetrically in branch 2, the rearranged link group denoted as $N_{1}-\mathrm{R}_{12}-\mathrm{R}_{2}-\mathrm{R}_{22}-N_{2}-\mathrm{R}_{23}-\mathrm{R}_{5}-\mathrm{R}_{32}-N_{3}-\mathrm{R}_{33}-\mathrm{R}_{3^{-}}$ $\mathrm{R}_{13}-N_{1}$ in Figure 6 is modified to be a movable elementary unit, and used to splice the 
novel coupled model. It is known that the numbers of edges and vertexes contained in a polyhedral solid satisfy the following Euler formula

$$
V+F-E=2
$$

where $V$ is the number of vertexes, $F$ is the number of faces and $E$ is the number of edges.

The relationship of $V=27, F=11$ and $E=36$ can be calculated for the coupled model, which satisfies Eq. (1) and demonstrates the reasonability of the novel mechanism.

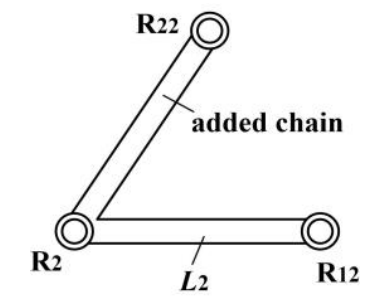

Figure 5. Adding Chain to Branch 1

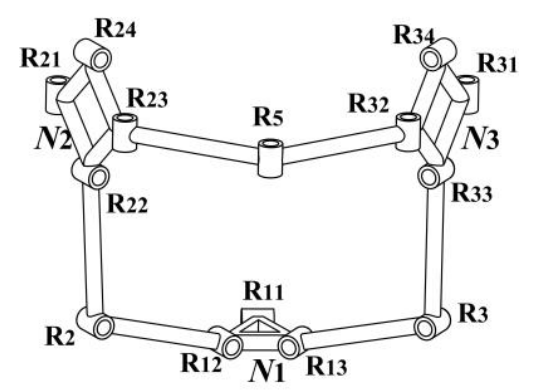

Figure 6. Moveable Elementary Loop

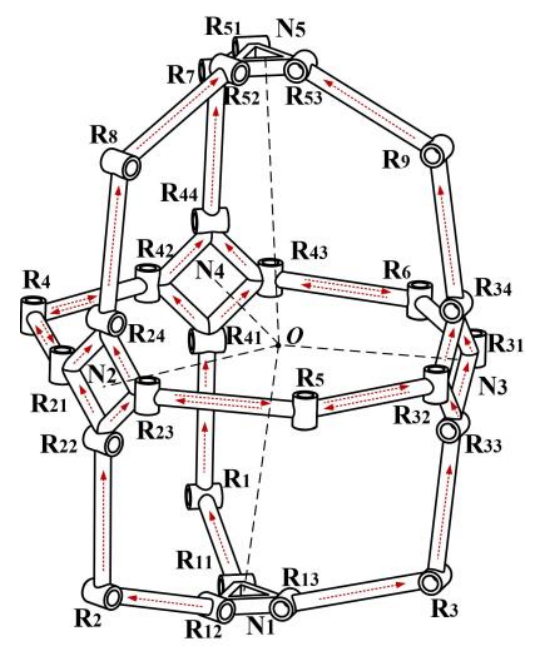

Figure 7. Structure of Novel Mechanism

\subsection{Structure of the Novel Coupled Mechanism}

As shown in Figure 7, the novel coupled model consists of two regular triangular components, i.e. $N_{1}$ and $N_{5}$, and three square nodes, i.e. $N_{2}, N_{3}$ and $N_{4}$, which are connected by 18 binary links with $27 \mathrm{R}$-pairs. $\mathrm{R}_{i 1} \sim \mathrm{R}_{i 3}(i=1,5)$ are the R-pairs located on $N_{i}$, and those fixed on $N_{j}$ are labeled as $\mathrm{R}_{j 1} \sim \mathrm{R}_{j 4}(j=2,3,4)$. The remainder nine mid-joints are $\mathrm{R}_{q}(q=1 \sim 9)$. The five lines, which are the normal to the plane of $N_{w}(w=1 \sim 5)$ and pass 
through their own geometrical centers, intersect at the point $O$. Each elementary loop is symmetrical to the plane passing through the axis of the mid-joint $\mathrm{R}_{q}$ and point $O$. The initial configuration for the following mobility analysis is represented by the mechanism configuration at any time of the moving process in Figure 7.

\section{Mobility Analysis of the Novel Coupled Mechanism}

Mobility analysis is the essential problem to recognize a new mechanism. The mobility of the proposed coupled mechanism is studied with the method of independent motion shunting measurement [19]. First, the coupled connections are split into three sub-chains according to the criterion of motion shunting. Then the relative motion of the nodes with respect to the base is expressed as generalized kinematic chains. Finally, the complicated coupled mechanism can be converted to an equivalent parallel mechanism. The mobility analysis of the novel mechanism is illustrated in detail as follows.

\subsection{Decomposition of the Novel Coupled Mechanism}

Referring to the Figure 7, the coupling chains connecting $N_{1}$ and $N_{5}$ are split into three identical branch $i(i=1,2,3)$. Each branch contains a coupled node, and the connection between the base and the coupled node is denoted as $C_{i}$. Every chain $C_{i}$ includes three sub-chains, i.e. $C_{i 1}, C_{i 2}$ and $C_{i 3}$ respectively, so $\mathrm{C}_{\mathrm{i}}$ is expressed as $C_{i}=C_{i 1} \cup C_{i 2} \cup C_{i 3}$ and formed to symmetrical double loops. In addition, three R-pairs are located between the coupled node and $N_{5}$, and any branch $i$ can be expressed as a chain of $C_{i}-\mathrm{R}^{\prime}-\mathrm{R}_{(i+6)}-\mathrm{R}_{5 i}$. In Figure 7 the motion flow of every branch is marked with red arrows and the corresponding sketch diagram is shown in Figure 8.

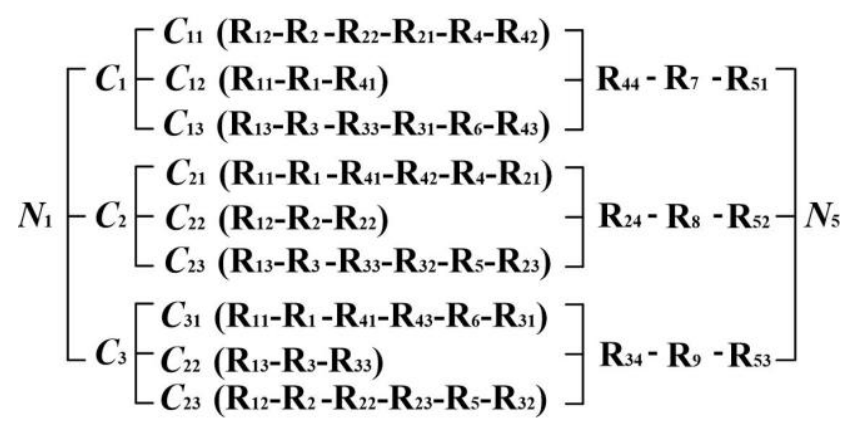

Figure 8. Sketch Diagram of Three Branches

\subsection{Mobility Analysis of the Chain C2}

As illustrated in Figure 8, the three parallel sub-chains in initial configuration can be labelled as $C_{21}\left(-\mathrm{R}_{11^{-}}-\mathrm{R}_{1}-\mathrm{R}_{41^{-}}-\mathrm{R}_{42^{-}}-\mathrm{R}_{4}-\mathrm{R}_{21^{-}}\right), C_{22}\left(-\mathrm{R}_{12}-\mathrm{R}_{2}-\mathrm{R}_{22^{-}}\right)$and $C_{23}\left(-\mathrm{R}_{13^{-}}-\mathrm{R}_{3}-\mathrm{R}_{33^{-}}-\mathrm{R}_{32}-\mathrm{R}_{5^{-}}\right.$ $\left.\mathrm{R}_{23^{-}}\right)$. $C_{21}$ and $C_{23}$ are located with a symmetrical plane perpendicular to $\mathrm{R}_{12^{-}}, \mathrm{R}_{2^{-}}$and $\mathrm{R}_{22^{-}}$ axes in branch 2. To analyze the constraints of each sub-chain, a local coordinate system $O_{1}-X_{1} Y_{1} Z_{1}$ in Figure 9 is built, where $Z_{1}$-axis is along the direction of $\mathrm{R}_{2}$-axis and the plane $X_{1} O_{1} Y_{1}$ coincides with the plane of symmetry in branch 2 aforementioned. Suppose that the intersection point of $\mathrm{R}_{22^{-}}$and $\mathrm{R}_{23}$-axes is denoted as $A_{1}\left(l_{1}, m_{1}, n_{1}\right)$ and the intersection point of $\mathrm{R}_{32^{-}}$and $\mathrm{R}_{33^{-}}$-axes as $A_{2}\left(l_{2}, m_{2}, n_{2}\right)$, the midpoint of $\mathrm{R}_{3}$-axis can be expressed as $A_{3}\left(a_{1}, 0, c_{1}\right)$ in $O_{1}-X_{1} Y_{1} Z_{1}$. 


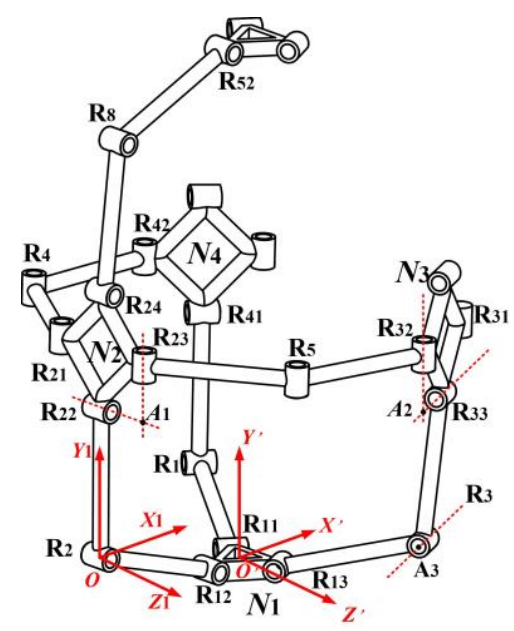

Figure 9. Coordinate System for Mobility Analysis of Branch 2

The screw system of the chain $C_{22}$ in initial configuration is

$$
\left\{\begin{array}{l}
\boldsymbol{S}_{12}=\left(\begin{array}{llllll}
0 & 0 & 1 ; & d_{1} & e_{1} & 0
\end{array}\right) \\
\boldsymbol{S}_{2}=\left(\begin{array}{llllll}
0 & 0 & 1 ; & 0 & 0 & 0
\end{array}\right) \\
\boldsymbol{S}_{22}=\left(\begin{array}{llllll}
0 & 0 & 1 ; & m_{1} & -l_{1} & 0
\end{array}\right)
\end{array}\right.
$$

where $d_{1}$ and $e_{1}$ represent two elements of dual unit of $\boldsymbol{S}_{12}$. The two symbols are just related to the axis location of R-pairs and do not depend on the mobility and constraint analysis of the mechanism. The subscripts of $\boldsymbol{S}_{12}, \boldsymbol{S}_{2}$ and $\boldsymbol{S}_{22}$ are related to that of the corresponding R-pairs.

Then the constraint screw system is obtained

$$
\left\{\begin{array}{l}
\boldsymbol{S}_{22}^{1 r}=\left(\begin{array}{llllll}
0 & 0 & 1 ; & 0 & 0 & 0
\end{array}\right) \\
\boldsymbol{S}_{22}^{2 r}=\left(\begin{array}{llllll}
0 & 0 & 0 ; & 1 & 0 & 0
\end{array}\right) \\
\boldsymbol{S}_{22}^{3 r}=\left(\begin{array}{llllll}
0 & 0 & 0 ; & 0 & 1 & 0
\end{array}\right)
\end{array}\right.
$$

which indicates a constraint force $\boldsymbol{S}_{22}^{1 r}$ and two constraint couples $\boldsymbol{S}_{22}^{2 r}$ and $\boldsymbol{S}_{22}^{3 r} \boldsymbol{S}_{22}^{1 r}$ can limit the translation along the $\mathrm{R}_{2}$-axis. $\boldsymbol{S}_{22}^{2 r}$ and $\boldsymbol{S}_{22}^{3 r}$ restrict the rotation of $N_{2}$ around the direction perpendicular to the $X_{1}$-axis and $Y_{1}$-axis.

The screw system of $C_{23}$ is expressed as

$$
\left\{\begin{array}{l}
\boldsymbol{S}_{13}=\left(\begin{array}{llllll}
a_{1} & 0 & c_{1} ; & d_{2} & e_{2} & f_{2}
\end{array}\right) \\
\boldsymbol{S}_{3}=\left(\begin{array}{llllll}
a_{1} & 0 & c_{1} ; & 0 & e_{3} & 0
\end{array}\right) \\
\boldsymbol{S}_{33}=\left(\begin{array}{llllll}
a_{1} & 0 & c_{1} ; & c_{1} m_{2} & a_{1} n_{2}-c_{1} l_{2} & -a_{1} m_{2}
\end{array}\right) \\
\boldsymbol{S}_{32}=\left(\begin{array}{llllll}
0 & 1 & 0 ; & -n_{2} & 0 & l_{2}
\end{array}\right) \\
\boldsymbol{S}_{5}=\left(\begin{array}{llllll}
0 & 1 & 0 ; & d_{4} & 0 & f_{4}
\end{array}\right) \\
\boldsymbol{S}_{23}=\left(\begin{array}{llllll}
0 & 1 & 0 ; & -n_{1} & 0 & l_{1}
\end{array}\right)
\end{array}\right.
$$

The constraint screw system will be

$$
\boldsymbol{S}_{23}^{1 r}=\left(\begin{array}{llllll}
0 & 0 & 0 ; & -c_{1} & 0 & a_{1}
\end{array}\right)
$$

Based on Eq. (4), there is a passive DOF in chain $C_{23}$. Eq. (5) states a constraint couple limiting the rotation of $N_{2}$ around the normal line of the plane defined by the $\mathrm{R}_{32^{-}}$and $\mathrm{R}_{33^{-}}$ axes. 
The constraint screw system of chain $C_{21}$ connected with the same structure as chain $C_{23}$, can be obtained symmetrically:

$$
\boldsymbol{S}_{21}^{1 r}=\left(\begin{array}{llllll}
0 & 0 & 0 ; & c_{1} & 0 & a_{1}
\end{array}\right)
$$

Solving the reciprocal screw system of the common constraint of Eq.(3), Eq.(5) and Eq.(6), we obtain

$$
\boldsymbol{S}_{2}^{g}=\left(\begin{array}{cccccc}
0 & 0 & 0 ; & 1 & 0 & 0 \\
0 & 0 & 0 ; & 0 & 1 & 0
\end{array}\right)
$$

which indicates the secondary reciprocal screw system of the symmetrical-double-loop and illustrates two translations of $N_{2}$ relative to the base.

\subsection{Mobility Analysis of C2-R24-R8-R52}

The motion of $C_{2}$ can be equivalent to two prismatic pairs, hence, branch 2 can be expressed as a generalized kinematic chain $\mathrm{P}_{1}-\mathrm{P}_{2}-\mathrm{R}_{24}-\mathrm{R}_{8}-\mathrm{R}_{52^{-}}$and the motion screw system in $O_{1}-X_{1} Y_{1} Z_{1}$ is

$$
\left\{\begin{array}{l}
\boldsymbol{S}_{\mathrm{p} 1}=\left(\begin{array}{llllll}
0 & 0 & 0 ; & 1 & 0 & 0
\end{array}\right) \\
\boldsymbol{S}_{\mathrm{p} 2}=\left(\begin{array}{llllll}
0 & 0 & 0 ; & 0 & 1 & 0
\end{array}\right) \\
\boldsymbol{S}_{24}=\left(\begin{array}{llllll}
0 & 0 & 1 ; & d_{5} & e_{5} & 0
\end{array}\right) \\
\boldsymbol{S}_{8}=\left(\begin{array}{llllll}
0 & 0 & 1 ; & d_{6} & e_{6} & 0
\end{array}\right) \\
\boldsymbol{S}_{52}=\left(\begin{array}{llllll}
0 & 0 & 1 ; & d_{7} & e_{7} & 0
\end{array}\right)
\end{array}\right.
$$

The constraint screw system of the equivalent chain is

$$
\boldsymbol{S}_{2}^{r}=\left(\begin{array}{cccccc}
0 & 0 & 1 ; & 0 & 0 & 0 \\
0 & 0 & 0 ; & 1 & 0 & 0 \\
0 & 0 & 0 ; & 0 & 1 & 0
\end{array}\right)
$$

which represents a constraint force limiting the translation along the $Z_{1}$-axis and two constraint couples restricting the rotation of $N_{5}$ around the direction of $X_{1}$-axis and $Y_{1}$-axis respectively. Based on Eq.(8) and (9) there are two passive degrees of freedom in each branch.

$$
\left\{\begin{array}{l}
\boldsymbol{S}_{1}^{1 r}=\left(\begin{array}{llllll}
1 & 0 & 1 ; & p_{1} & q_{1} & r_{1}
\end{array}\right) \\
\boldsymbol{S}_{1}^{2 r}=\left(\begin{array}{llllll}
0 & 0 & 0 ; & p_{2} & q_{2} & r_{2}
\end{array}\right) \\
\boldsymbol{S}_{1}^{3 r}=\left(\begin{array}{llllll}
0 & 0 & 0 ; & p_{3} & q_{3} & r_{3}
\end{array}\right) \\
\boldsymbol{S}_{2}^{1 r}=\left(\begin{array}{llllll}
0 & 0 & 1 ; & 0 & 0 & 0
\end{array}\right) \\
\boldsymbol{S}_{2}^{2 r}=\left(\begin{array}{llllll}
0 & 0 & 0 ; & 0 & q_{5} & 0
\end{array}\right) \\
\boldsymbol{S}_{2}^{3 r}=\left(\begin{array}{llllll}
0 & 0 & 0 ; & p_{6} & 0 & 0
\end{array}\right) \\
\boldsymbol{S}_{3}^{1 r}=\left(\begin{array}{llllll}
1 & 0 & 1 ; & 0 & q_{7} & 0
\end{array}\right) \\
\boldsymbol{S}_{3}^{2 r}=\left(\begin{array}{llllll}
0 & 0 & 0 ; & p_{8} & q_{8} & r_{8}
\end{array}\right) \\
\boldsymbol{S}_{3}^{3 r}=\left(\begin{array}{llllll}
0 & 0 & 0 ; & p_{9} & q_{9} & r_{9}
\end{array}\right)
\end{array}\right.
$$

\subsection{Mobility Analysis of the Novel Coupled Mechanism}

To describe the overall constraints acting on $N_{5}$, the global coordinate system $O^{\prime}$ $X^{\prime} Y^{\prime} Z^{\prime}$ is established shown in Figure 9, where the origin $O^{\prime}$ is the projection of the geometrical center $O$ on the base $N_{1}$ and the three axes of $O^{\prime}-X^{\prime} Y^{\prime} Z^{\prime}$ are parallel to that of the coordinate system $O_{1}-X_{1} Y_{1} Z_{1}$ respectively. The other two branches have the similar 
characteristics as that of branch 2 for symmetry, therefore, the constraint screw system of all branches can be expressed in $O^{\prime}-X^{\prime} Y^{\prime} Z^{\prime}$ as

The secondary reciprocal screw system is

$$
\boldsymbol{S}^{r r}=\left(\begin{array}{llllll}
0 & 0 & 0 ; & 0 & 1 & 0
\end{array}\right)
$$

which indicates that $N_{5}$ has a translation DOF along $Y_{1}$-axis, which also demonstrates the reciprocal motion of the moving plane.

\subsection{Mobility Analysis of the Equivalent Parallel Mechanism}

Based on the mobility analysis described above, the novel coupled mechanism can be equivalent to the parallel mechanism shown in Figure 10. Each generalized chain provides a constraint force and two constraint couples. There is a common constraint $(\lambda=1)$ among these constraint couples and the order of the mechanism is five $(d=6-1=5)$; the rank of the three linearly dependent constraint forces is two. There are two parallel redundant constraints and six passive degrees of freedom overall. According to the modified G-K formula, the DOF of the mechanism is solved [21]

$$
M=d(n-g-1)+\sum_{i=1}^{g} f_{i}+v-\zeta=1
$$

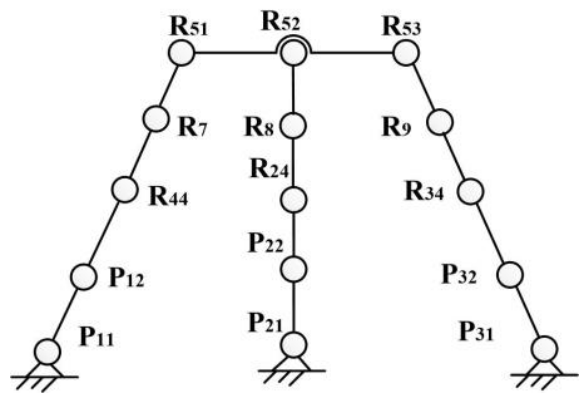

Figure 10. Equivalent Parallel Mechanism

The relationship of the relative motion and constraints characteristic of the three branches keep invariant in any configuration, so we can always set up the local and global coordinates systems in Figure 9. Thus the kinematic screw systems and constraint screw systems maintain the same. It is proved that the mobility analysis of the coupled mechanism is full-cycle.

\section{Motion Simulation}

The motion characteristic of the novel coupled mechanism is simulated in this section. The simulation model is established first and then the spatial displacement variations of the moving plane and three nodes are analyzed. The correctness of theoretical mobility analysis and motion properties of the novel mechanism is illustrated by the curves of simulating results finally.

\subsection{Simulation Model}

The simulation model established by SolidWorks is shown in Figure 11 and the Table 2 illustrates the design parameters for the three types of substitutive components respectively. The symbol $l_{i}$ indicates the side-length of the corresponding component and $r_{j}$ expresses the radius of axis-hole for R-pairs. 


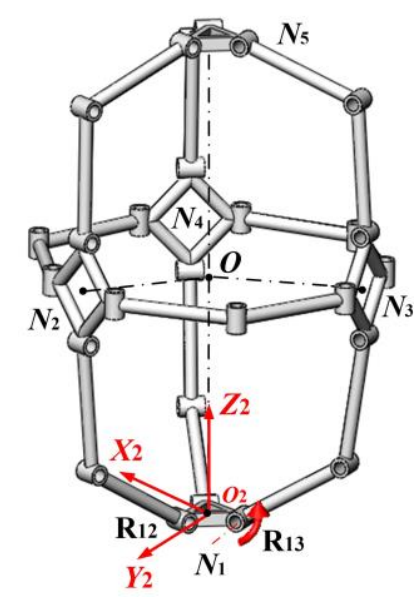

Figure 11. Simulation Model

Table 2. Design Parameters of the Three Substitutive Parts (Mm)

\begin{tabular}{|c|c|c|}
\hline Components & Parameters & Size \\
\hline \multirow{2}{*}{ regular triangle } & $l_{1}$ & 9 \\
\cline { 2 - 3 } & $r_{1}$ & 1 \\
\hline \multirow{2}{*}{ binary link } & $l_{2}$ & 20 \\
\hline \multirow{2}{*}{ square node } & $r_{2}$ & 1 \\
\cline { 2 - 3 } & $l_{3}$ & 9 \\
\hline
\end{tabular}

To realize one translation for $N_{1}$, a rotational motor at $10 \mathrm{rpm}$ shown with a red curved arrow in Figure 11 is set on $\mathrm{R}_{13}$-axis. A coordinate system $O_{2}-X_{2} Y_{2} Z_{2}$ is established to determine the position variations of the end-effector and nodes. The origin $\mathrm{O}_{2}$ located in the center of the base $N_{1}$. The $X_{2}$-axis is parallel to the direction of $\mathrm{R}_{12}$-axis. The plane of $\mathrm{X}_{2} \mathrm{O}_{2} Y_{2}$ is parallel to $\mathrm{N}_{1}$ and $\mathrm{Y}_{2} \mathrm{O}_{2} \mathrm{Z}_{2}$ coincides with the symmetrical plane of branch 2 .

\subsection{Simulation of Displacement Variations}

The output motion along three axes is simulated with the center of $N_{5}$ and the displacement variations of the three nodes are implemented by the three centers of $N_{2}, N_{3}$ and $N_{4}$ respectively in the global system $\mathrm{O}_{2}-\mathrm{X}_{2} Y_{2} \mathrm{Z}_{2}$. The range of the displacement of $N_{5}$ is from $74.9693 \mathrm{~mm}$ to $94.7397 \mathrm{~mm}$ and the corresponding motion simulation of the four centers along the three axes are conducted by running the motor for $2 \mathrm{~s}$, divided into 51 steps each lasting $0.04 \mathrm{~s}$.

To display the mobility of the end, time-displacement curve of $N_{5}$ along the three axes is plot in Figure 12. 


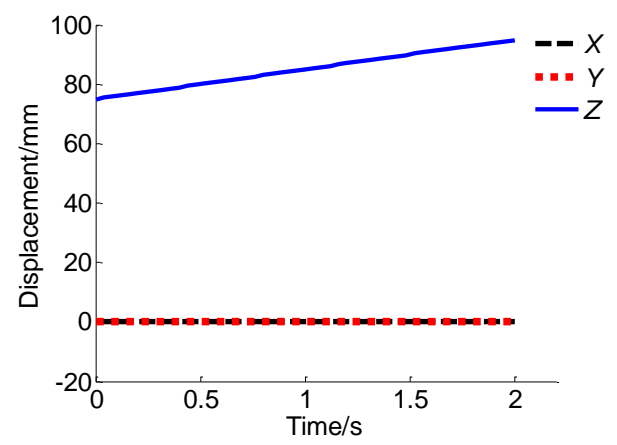

Figure 12. Motion Simulation of N5 along X-, Y- and Z-Axis

Figure 12 shows that the translation along $X$-axis (black dashed line) and $Y$-axis (red dotted line) is unchanged with time, and the translation of $Z$-axis (blue solid line) rises gently. Hence, the end-effector just has one translation along $Z$-axis and the correctness of theoretical mobility analysis in Section 3 is demonstrated.

To highlight the motion characteristics of $N_{2}, N_{3}$ and $N_{4}$, the displacement of each node along the same axis is plot in a graph simultaneously for comparison. The contrastive time-displacement curves along three axes are shown in Figure 13 Figure 15 respectively. The corresponding spatial displacement curves of the three nodes and moving plane is plot in Figure 16.

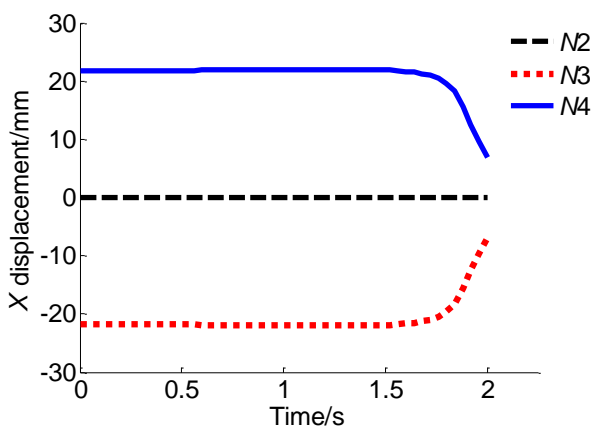

Figure 13. Motion Simulation of N2, N3 and N4 along X-axis

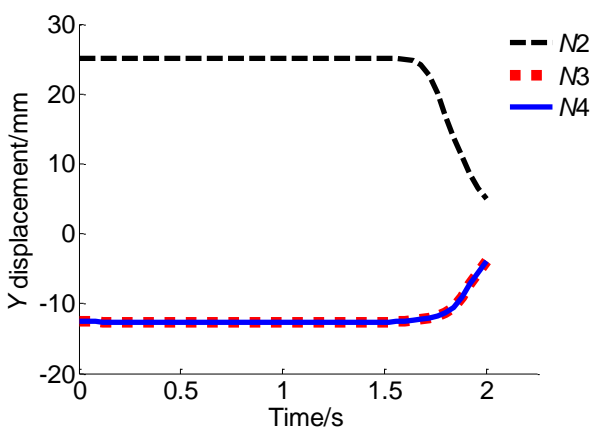

Figure 14. Motion Simulation of N2, N3 and N4 along Y-axis 


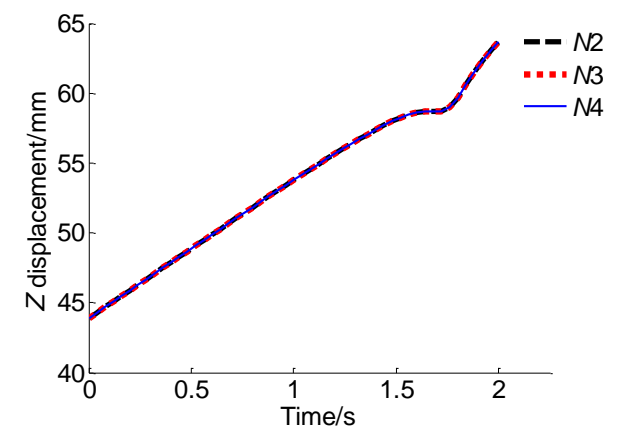

Figure 15. Motion Simulation of N2, N3 and N4 along Z-axis

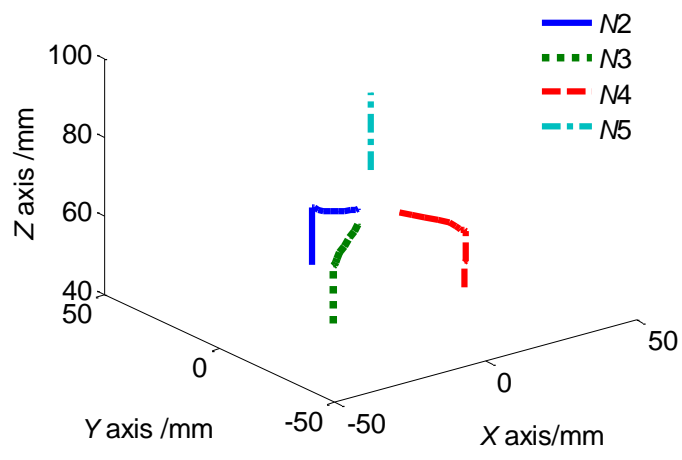

Figure 16. Spatial Displacement Variations for Parts

The comparison of the curves shown in Figure 13 Figure 15 demonstrates that the motion of $N_{2}$ is confined in the plane $\mathrm{XOZ}$, which also represents the plane of symmetry of branch 2. The variations of the three nodes along $Z$-axis keep identical during the whole simulation. Figure 16 means that $N_{2}, N_{3}$ and $N_{4}$ exhibit good characteristics of radial motion tendency towards to the center $O$ with associated reciprocal translation of $N_{5}$ along $Z$-axis.

\section{Results and Discussion}

Above relationships satisfy the mobility analysis in Section 3 and illustrate the properties of symmetry of the novel coupled mechanism. The coupled mechanism has one translational DOF and the three nodes move symmetrically with the radial motion tendency directing to the geometrical center.

Based on the above simulations, the proposed mechanism synthesized symmetrically is a deployable mechanism. The expandable and retractable configurations of the moving plane relative to the base are shown in Figure 17. 


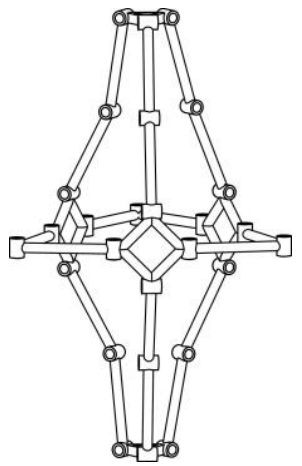

(a) Retractable Configuration

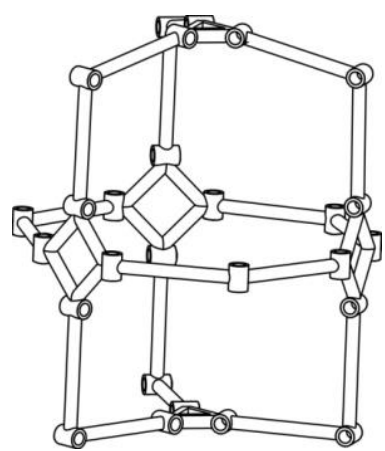

(b) Expandable Configuration

Figure 17. Configuration of the Deployable Mechanism

\section{Conclusions}

A novel symmetrical coupled mechanism based on a compound of two regular tetrahedrons is proposed. The coupled structure is obtained by designing three types of substitutive parts for edges and vertexes. The movable elementary unit is modified by adding chains symmetrically. The mobility analysis based on the screw theory demonstrates the new coupled mechanism with a translational DOF is feasible.

A simulation model is established to show the motion characteristics of the components. According to the displacement variation of the end and nodes, the coupled mechanism is proved a deployable mechanism with one reciprocal translation accompanied by radial moving tendency of nodes towards the geometrical center. The three nodes always arranged to the symmetrical plane of the moveable elementary loop.

The novel mechanism proposed in this paper may be considered as an execution structure of deployable mobile robots and paves the way for developing more kinds of polyhedral coupled mechanisms.

\section{Acknowledgements}

This paper was supported by the NSFC (Grant No. 51475015) and the National Engineering Laboratory for Highway Maintenance Equipment (Grant No. 3108251 51130).

\section{References}

[1] Feng Gao. Reflection on the Current Status and Development Strategy of Mechanism Research. Journal of Mechanical Engineering. Vol. 41, No. 8, p. 4-17. (2005)

[2] Huiping Shen, Haibin Zhao, Jiaming Deng, Qingmei Meng, Wei Zhu, Tingli Yang. Type Design Method and the Application for Hybrid Robot Based on Freedom Distribution and Position and Orientation Characteristic set. Journal of Mechanical Engineering. Vol. 47, No. 23, p. 56-64. (2011)

[3] L. Tsai, S. Joshi. Kinematic Analysis of 3-DOF Position Mechanisms for Use in Hybrid Kinematic Machines. Journal of Mechanical Design. Vol. 124, No. 2, p. 245-253. (2002)

[4] Ju Li, Dean Zhao, Huiping Shen, Jiaming Deng, Yixing Jiang, Wei Xu. Design of 5-axes Hybrid Robot with Several Spray Guns for Collaborative Spraying. Transactions of the Chinese Society for Agricultural Machinery. Vol. 43, No. 4, p. 216-220. (2012)

[5] J. M. Hervé. The Lie Group of Rigid Body Displacements, A Fundamental Tool for Mechanism Design. Mechanism and Machine Theory. Vol. 34, No. 5, p. 719-730. (1999)

[6] Qiang Zeng, Yuefa Fang. Structural Synthesis and Analysis of Serial-Parallel Hybrid Mechanisms with Spatial Multi-loop Kinematic Chains. Mechanism and Machine Theory. Vol. 49, p. 198-215. (2012)

[7] Qinchuan Li, Zhen Huang. Type Synthesis of 3R2T 5-DOF Parallel Manipulators Using the Lie Group of Displacements. IEEE Transactions on Robotics and Automation. Vol. 20, No. 2, p. 173-180. (2004)

[8] Guowu Wei, Xilun Ding, Jiansheng Dai. Geometric and Kinematic Analysis of the Hoberman SwitchPitch Ball and its Variant. Proceedings of the ASME International Design Engineering Technical 
Conferences and Computers and Information in Engineering Conference, (2009), August, August 30September 2, San Diego, California, USA, PARTS A AND B, p. 1245-1254.

[9] Zhen Huang, Qinchuan Li. Type Synthesis of Symmetrical Lower-mobility Parallel Mechanisms using Constraint-Synthesis Method. The International Journal of Robotics Research. Vol. 22, No. 1, p. 59-79. (2003)

[10] Zhen Huang, Jingfang Liu, Daxing Zeng. A General Methodology for Mobility Analysis of Mechanisms Based on Constraint Screw Theory. Science in China, Series E: Technological Sciences. Vol. 52, No. 5, p. 1337-1347. (2009)

[11] Z. You, S. Pellegrino. Foldable Bar Structure. International Journal of Solids and Structures. Vol. 34, No. 15, p. 1825-1847. (1997)

[12] L.Thierry, C. Gosselin. Construction, Mobility Analysis and Synthesis of Polyhedra with Articulated Faces. Journal of Mechanisms and Robotics. Vol. 6, No. 1, 011007. (2013)

[13] Yezhuo Li, Yaobin Tian, Yan'an Yao. Foldable Triangular Bipyramid Rolling Mechanism. Journal of Mechanical Engineering. Vol. 50, No. 11, p. 68-78. (2014)

[14] Zheng Liu, Yichao Chen, Huafeng Ding. Kinematics Analysis of a New Hydraulic Face-shovel Excavator in Mining. Journal of Mechanical Engineering. Vol. 50, No. 15, p. 28-33. (2014)

[15] A. Campos, C.Budde, J. Hesselbach. A Type Synthesis Method for Hybrid Robot Structures. Mechanism and Machine Theory. Vol. 43, No. 8, p. 984-995. (2008)

[16] Jiansheng Dai, J. Jones. Mobility in Metamorphic Mechanisms of Foldable/Erectable Kinds. Transactions of ASME: Journal of Mechanical Design. Vol. 121, No. 3, p. 375-382. (1999)

[17] Jiansheng Dai, Duanling Li, Qixian Zhang, Guoguang Jin. Mobility Analysis of a Complex Structured Ball Based on Mechanism Decomposition and Equivalent Screw System Analysis. Mechanism and Machine Theory. Vol. 39, No. 4, p. 445-458. (2004)

[18] Z. You. Motion Structures Extend Their Reach. Materials Today. Vol. 10, No. 12, p. 52-57. (2007)

[19] Jingfang Liu, Xiaoou Huang, Yueqing Yu, Huafeng Ding, Zhen Huang. Equivalent Method of Output Mobility Calculation for a Novel Multi-loop Coupled Mechanism. Journal of Mechanical Engineering. Vol. 50, No. 23, p. 13-19. (2014)

[20] Huifang Gao, Jingfang Liu, Xiaoou Huang. Method of Mobility Calculation for a Coupled Mechanism Based on Independent Motion Shunting Measurement. Journal of Beijing University of Technology. Vol. 41, No. 11, p. 1658-1664. (2015)

[21] Zhen Huang, Jingfang Liu, Yanwen Li. Theories on Degree of Freedom of Mechanism. Beijing: Science and Education Press, p. 84+93. (2011)
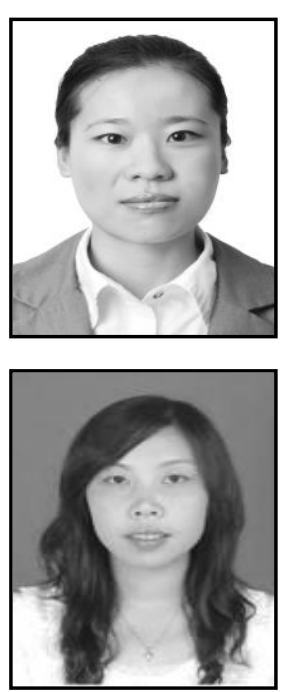

\section{Authors}

Gao Huifang, born in 1988 , is currently a $\mathrm{PhD}$ candidate at College of Mechanical Engineering and Applied Electronics Technology, Beijing University of Technology, China. Her research interests include synthesis and analysis for spatial mechanisms.

Liu Jingfang, born in 1985, is currently an associate professor at College of Mechanical Engineering and Applied Electronics Technology, Beijing University of Technology, China. She received her $\mathrm{PhD}$ degree from Yanshan University, China. Her research interests include synthesis and analysis for spatial mechanisms. 